\title{
Falls prevention interventions for community-dwelling older people living in mainland China: a narrative systematic review
}

Pengpeng Y Y $e^{1,2^{*}+} \mathbb{D}$, Yishu Liu ${ }^{1,3+}$, Jing Zhang ${ }^{4}$, Ke Peng $^{1,5}$, Xuru Pan ${ }^{6}$, Yang Shen ${ }^{3}$, Shaoming Xiao ${ }^{6}$, Elizabeth Armstrong ${ }^{4,7}$, Yuliang Er ${ }^{2}$, Leilei Duan², Rebecca Ivers ${ }^{1,4}$, Lisa Keay ${ }^{1,8}$ and Maoyi Tian ${ }^{1,3}$

\begin{abstract}
Background: Falls in community-dwelling older people have been recognised as a significant public health issue in China given the rapidly growing aged population. Although there are several reviews documenting falls prevention programs for community-dwelling older adults, no systematic reviews of the scope and quality of falls prevention interventions in Mainland China exist. Therefore, the aim of this study was to systematically review falls prevention interventions for community-dwelling older people living in Mainland China.

Methods: We systematically reviewed literature from Chinese and English databases. All types of randomised controlled trials (RCTs) and quasi-experimental studies published from 1st January 1990 to 30th September 2019 were included. Observational studies and studies in care facilities and hospitals were excluded. Narrative synthesis was performed to summarise the key features of all included studies. Quality assessment was conducted using the Cochrane Risk of Bias Tool and ROBINS-I tool for randomised and non-randomised studies respectively.

Results: A total of 1020 studies were found, and 101 studies were included in the analysis. Overall, very few high quality studies were identified, and there was insufficient rigor to generate reliable evidence on the effectiveness of interventions or their scalability. Most interventions were multiple component interventions, and most studies focused on outcomes such as self-reported falls incidence or awareness of falls prevention.

(Continued on next page)
\end{abstract}

\footnotetext{
* Correspondence: pye@georgeinstitute.org.au

${ }^{\dagger}$ Pengpeng Ye and Yishu Liu contributed equally to this work.

${ }^{1}$ The George Institute for Global Health, University of New South Wales, PO Box M201, Missenden Road, Sydney, NSW 2050, Australia

${ }^{2}$ National Centre for Non-Communicable Disease Control and Prevention, Chinese Centre for Disease Control and Prevention, Beijing, China Full list of author information is available at the end of the article
}

(C) The Author(s). 2020 Open Access This article is licensed under a Creative Commons Attribution 4.0 International License, which permits use, sharing, adaptation, distribution and reproduction in any medium or format, as long as you give appropriate credit to the original author(s) and the source, provide a link to the Creative Commons licence, and indicate if changes were made. The images or other third party material in this article are included in the article's Creative Commons licence, unless indicated otherwise in a credit line to the material. If material is not included in the article's Creative Commons licence and your intended use is not permitted by statutory regulation or exceeds the permitted use, you will need to obtain permission directly from the copyright holder. To view a copy of this licence, visit http://creativecommons.org/licenses/by/4.0/ The Creative Commons Public Domain Dedication waiver (http://creativecommons.org/publicdomain/zero/1.0/) applies to the data made available in this article, unless otherwise stated in a credit line to the data. 


\begin{abstract}
(Continued from previous page)
Conclusion: There is an opportunity to undertake an evaluation of a rigorously-designed, large-scale falls prevention program for community-dwelling older people in Mainland China. To help mitigate the rising burden of falls in Mainland China, recommendations for future falls prevention interventions have been made. These include: (1) target disadvantaged populations; (2) incorporate personalised interventions; and (3) investigate the effectiveness of those under-explored interventions, such as psychological, social environment, management of urinary incontinence, fluid or nutrition therapy and surgery. The study results will also potentially provide a useful evidence base for other low-and-middle income countries in a similar situation.
\end{abstract}

Keywords: Falls prevention, Interventions, Older people, Community, China

\section{Background}

Falls, defined as 'events which result in a person coming to rest inadvertently on the ground or floor or other lower level' [1], are a major health issue for older adults [2]. Globally, about $30 \%$ of community dwelling people aged 65 years and over will fall at least once each year [2]. This number increases to $50 \%$ for those older than 85 years [2]. The Global Burden of Disease (GBD) Study 2017 estimated falls as the 11th cause of all-cause disability-adjusted life-years (DALYs) and they caused 32 million severe injuries and 400,000 deaths for people aged 70 years and above worldwide [3]. In China, falls are one of the leading causes of injury-related DALYs amongst older people in 2017 [4]. The GBD 2017 showed an increased incidence of falls and associated mortality and DALYs amongst older people in China over the past three decades [4].

Falls have been widely recognised as a complex but preventable health issue amongst older people [3]. A variety of community-based falls prevention interventions have been documented and evaluated in recent reviews [5-13]. Exercise interventions have been shown to be effective in reducing fear of falling and falls amongst community-dwelling older people $[8,9,13]$. The combination of exercise, education, medication modification and home environment improvements have also been found to be an effective packaged intervention [6, 7, 10-12]. However, most studies included in these reviews were conducted in high income countries. Few studies conducted in the Mainland China were synthesized in the previous reviews $[11,12]$. The scope and quality of falls prevention interventions for community-dwelling older people in Mainland China also remain unclear. Therefore, the aim of this study was to systematically review falls prevention interventions for community-dwelling older people in Mainland China, to help inform policy and practice in a country with a rapidly rising burden of falls.

\section{Methods}

\section{Protocol and registration}

The protocol of this systematic review was registered in PROSPERO (CRD42018085507). This review is reported according to the guidance of the Preferred Reporting Items for Systematic Review and Meta-analysis (PRIS MA) [14].

\section{Search strategy and study selection}

A systematic search of the literature in both Chinese and English published from 1st January 1990 to 30th September 2019 was performed using the following electronic databases: Medline, Excerpta Medica Database (Embase), Web of Science, Cumulative Index to Nursing and Allied Health Literature (CINAHL), Cochrane Library, Chinese National Knowledge Infrastructure (CNKI), Chinese Wanfang Database, China Biology Medicine disc (CMBdisc), and Chongqing VIP Database for Chinese Technical Periodical. The searched keywords included fall, accidental falls, aged, senior, elderly, old, older, community, intervention, prevention, China, and Chinese. A detailed search strategy is listed in Additional File 4. The reference lists of included studies were scanned for additional potential studies. Two independent reviewers (PY and YL) screened the search results according to the eligibility criteria. Disagreements were resolved through discussion and a third reviewer (JZ) where necessary.

\section{Eligibility criteria}

We included all articles reporting falls prevention interventions for community-dwelling older people in Mainland China. All types of randomised controlled trials (RCTs) and quasi-experimental studies with or without comparators were included. We only included studies satisfying the following criteria: (1) study participants aged 60 years and above with autonomous mobility or with adequate mobility when using assistive devices; and (2) conducted in community settings in Mainland China. We excluded studies conducted in care facilities or hospitals and/or non-peer reviewed research articles. There was no restriction applied to study outcomes.

\section{Data extraction}

A standardised data abstraction form was developed (Additional File 1) for entering key study characteristics, including language, location, area, year, study population, 
design, protocol, intervention duration, intervention type, comparator, outcome measurements, and results. Two reviewers (PY and YL) independently extracted the data into the form and cross-reviewed. Disagreements were resolved by consensus.

\section{Quality assessment}

For RCTs, methodological quality was assessed using the Cochrane Risk of Bias Tool [15]. We assessed the random sequence generation, allocation concealment, blinding of participants, personnel and outcomes assessors, incomplete outcome data, selective outcome reporting, and other sources of bias. Non-randomised trials were assessed by the ROBINS-I tool (Risk of Bias in Non-Randomised Studies - of Interventions) [16]. Any discrepancies were discussed and resolved by consensus (PY, YL and JZ).

\section{Data synthesis}

We utilised a slightly adapted classification of falls prevention interventions from Hopewell's Cochrane review [10]. In this review, the interventions were classified into three types (single intervention, multiple component intervention and multifactorial intervention) and nine categories (exercise, medication, surgery, management of urinary incontinence, fluid or nutrition therapy, psychological intervention, environment/assistive technology, social environment and knowledge/education). In each category, there were different intervention components (Additional File 2). Single intervention type is comprised of one or more intervention component from single intervention category alone. Multiple component intervention type provides a fixed combination of two or more fall prevention components from different intervention categories. Multifactorial intervention type provides people with an individualised combination of two or more components from different intervention categories based on assessment of modifiable risk factors. The study outcomes were categorized into four types based on the information extracted from all eligible studies, including fall-related injuries, fall incidence, fall-related physical performance and other outcomes related to falls (Additional File 3). A quantitative meta-analysis was not performed due to the heterogeneity of the included studies.

\section{Results}

\section{Study selection}

We retrieved 1020 articles from nine databases and 132 articles were selected for full-text review (Fig. 1). Of those, 31 articles were excluded and 101 articles were included in the final synthesis [17-117].

\section{Study characteristics}

The study characteristics are summarised in Additional File 1. All 101 studies were published after the year 2000 with 68 studies conducted since 2011. Studies were conducted across 21 Chinese provinces. Almost half of the studies were conducted in high socioeconomic regions: 18 studies in Shanghai municipality, 14 studies in Guangdong province, 10 studies in Zhejiang province, and 7 studies in Beijing municipality (Fig. 2). The majority of studies (97 studies) were conducted in urban settings

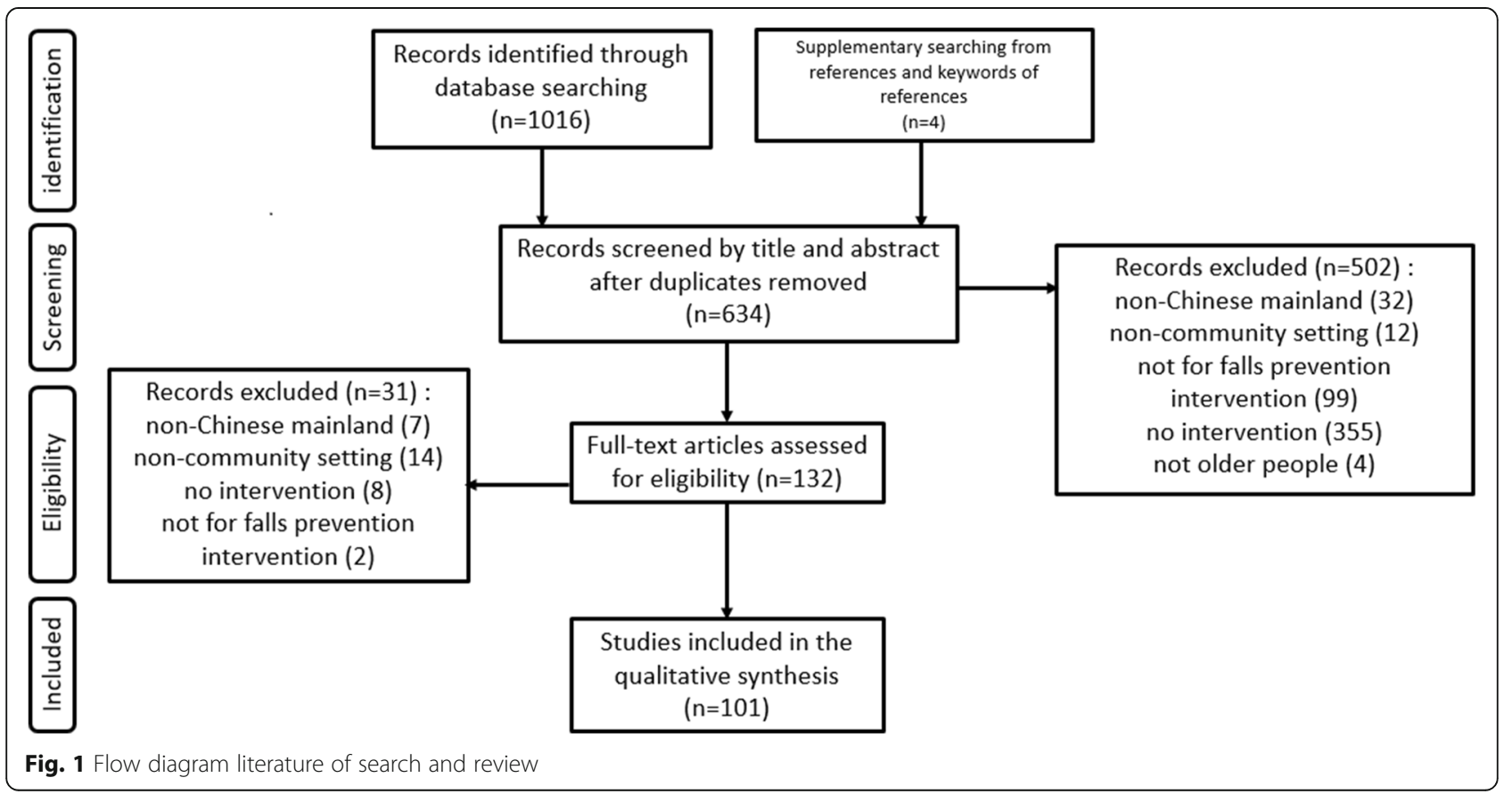




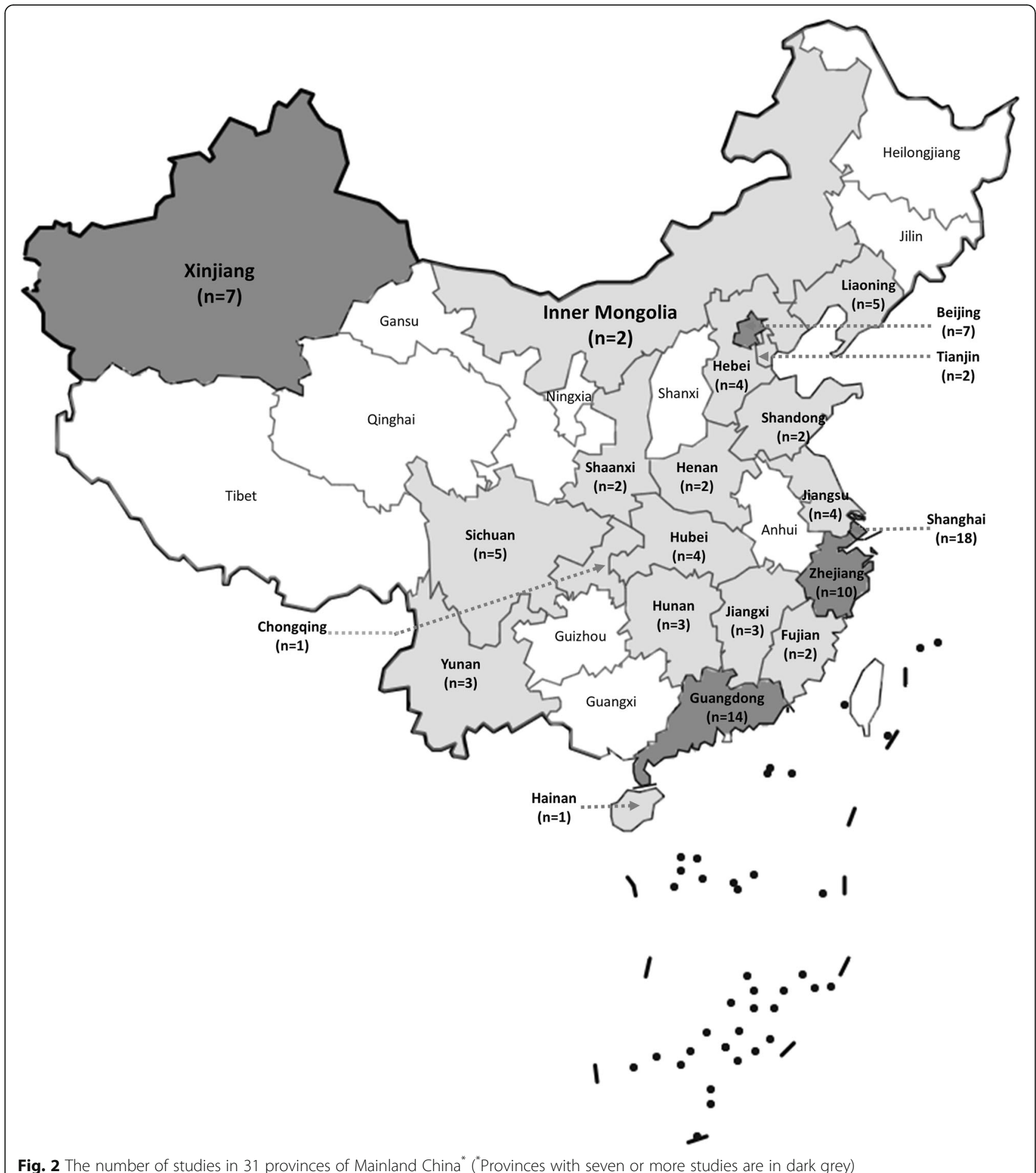

with only 4 studies focusing on a rural population. There were 77 studies with RCT design and 24 studies with quasi-experimental design. The sample size of the RCT studies varied from 22 participants to 2643 participants, and in the quasi-experimental studies, sample size varied from 16 participants to 3466 participants. Only 4 RCTs and 4 quasi-experimental studies had sample sizes of more than 500 participants.

Single interventions were tested in $17 \mathrm{RCTs}$ and 3 quasi-experimental studies, whereas multiple component interventions were tested in $47 \mathrm{RCTs}$ and 15 quasiexperimental studies. Multifactorial interventions were 
tested in 13 RCTs and 6 quasi-experimental studies (Table 1, Additional File 2). Most studies reported two or more outcomes. The majority of outcomes were falls per person and falls prevention awareness (Table 2, Additional File 3).

\section{Intervention types}

\section{Single intervention}

The most commonly used single intervention category (16 studies) was exercise. In this category, the two most commonly used components were gait exercise, balance and functional training (10 studies), and strength/resistance exercises (9 studies). Traditional Chinese Health Qigong as three-dimensional training component, e.g., Tai Chi and Ba Duan Jin, was also adopted in 7 studies. There was only one RCT reporting the effectiveness of homecare services from social environment category to prevent falls for older people. There were two RCTs and one quasi-experimental study that evaluated knowledge/ education intervention category (Additional File 2).

\section{Multiple component intervention}

In total, there were 63 studies utilising multiple component interventions. The number of intervention categories varied between two and six. Most studies (38 studies) adopted four or more categories. The combination of knowledge/education, exercise, environment/assistive technology and medication were the most commonly used package, adopted in 32 studies. The most common component for knowledge/education category was the distribution of health education written material (50 studies) and delivery of health lectures (49 studies). For exercise interventions, gait, balance and functional training (22 studies), strength/resistance exercises (11 studies) and three-dimensional training (11 studies) were usually adopted to prevent falls for older people. Furnishings and adaptations to homes and other premises (28 studies) were widely used in the environment/assistive technology category. There were 20 studies reporting the use of vitamin $\mathrm{D}$ and calcium supplementation as main components of medication category. As for the remaining five intervention categories, psychological and social environment were adopted in 15 and 5 studies, whereas surgery, management of urinary incontinence and fluid or nutrition therapy were not implemented in any of the included studies (Additional File 2).

\section{Multifactorial intervention}

Multifactorial interventions were the least commonly used intervention type (19 out of 101 studies). Up to 6 intervention categories were delivered to older people based on the assessment of their fall-related risk factors, including history of disease, history of falls, balance, fear of falling, home environment, and activities of daily living. The most commonly fixed combination of intervention categories was knowledge/education, environment/assistive technology and exercise. The main components in these three categories were very similar to those found in the multiple component intervention type, namely, the distribution of health education written material (16 studies) and delivery of health lectures (15 studies) in knowledge/education, furnishings and adaptations to homes and other premises (11 studies) in environment/assistive technology, and the gait, balance and functional training (3 studies) and three-dimensional training (3 studies) in exercise. Compared to the main components of the medication category in the multiple component type, the frequently used components of medication in multifactorial studies were medication withdrawal (4 studies) and dose reduction or increase, substitution or provision (3 studies). There were 9 studies reporting the use of a psychological intervention category, of which the most common component was an individual cognitive (behavioural) intervention (6 studies). Social environment category was utilized in 7 studies, of which staff training (5 studies) and service model change (studies) were the main components. Surgery, management of urinary incontinence and fluid or nutrition therapy categories were not adopted in any of the multifactorial intervention studies (Additional File 2).

\section{Outcomes}

There were no studies reporting fall-related mortality as the study outcome. Fall-related injuries as outcomes of effectiveness of interventions were rarely reported, only for fall-related fracture events (8 studies), hospital admissions due to falls ( 2 studies) and medical cost (1 study). Fallers per person as a preferred indicator of fall incidence were reported in 67 studies, which was much

Table 1 Number of studies by intervention type and study design

\begin{tabular}{|c|c|c|c|c|c|c|c|c|c|c|}
\hline \multirow[t]{2}{*}{ Study design } & \multicolumn{3}{|c|}{ Single intervention } & \multicolumn{3}{|c|}{ Multiple component intervention } & \multicolumn{3}{|c|}{ Multifactorial intervention } & \multirow[t]{2}{*}{ Tota } \\
\hline & Education & Exercise & $\begin{array}{l}\text { Social } \\
\text { environment }\end{array}$ & $\begin{array}{l}2-3 \\
\text { components }\end{array}$ & $\begin{array}{l}4-5 \\
\text { components }\end{array}$ & $\begin{array}{l}\geq 6 \\
\text { components }\end{array}$ & $\begin{array}{l}2-3 \\
\text { components }\end{array}$ & $\begin{array}{l}4-5 \\
\text { components }\end{array}$ & $\begin{array}{l}\geq 6 \\
\text { components }\end{array}$ & \\
\hline $\mathrm{RCT}$ & 2 & 14 & 1 & 16 & 30 & 1 & 6 & 6 & 1 & 77 \\
\hline Quasi-experimental & 1 & 2 & 0 & 8 & 7 & 0 & 3 & 2 & 1 & 24 \\
\hline Total & 3 & 16 & 1 & 24 & 37 & 1 & 9 & 8 & 2 & 101 \\
\hline
\end{tabular}




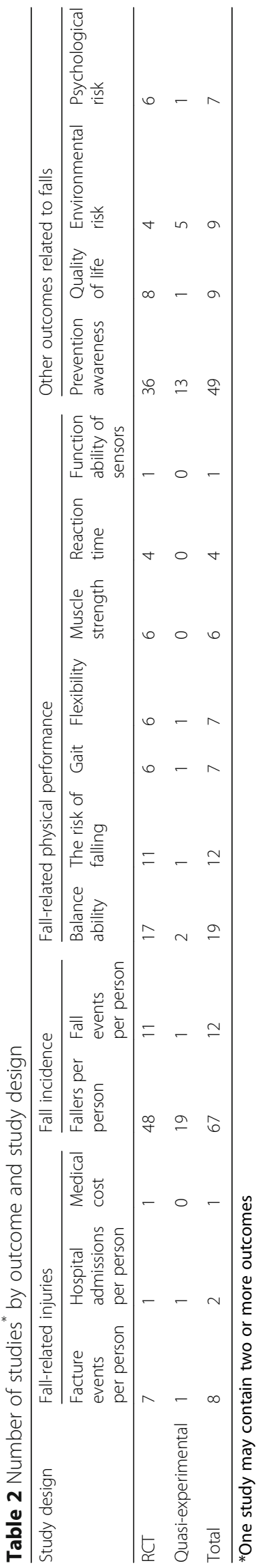


more than studies reporting fall events per person (12 studies). As for fall-related physical performance, balance was one of most relevant causes of falls and reported in 19 studies, followed by risk of falling (12 studies), gait (7 studies), flexibility (7 studies), muscle strength (6 studies), reaction time (4 studies), and function ability of sensors (1 study). Other outcomes associated with the risk of falls were reported in some studies, of which 49 studies evaluated the awareness of falls prevention (49 studies), followed by quality of life ( 9 studies), environmental risk (9 studies), and psychological risk (7 studies). A wide range of measurement tools were used to assess the effect of the intervention, including standardised scales, questionnaires and devices. Despite the heterogeneity of outcome measures, all studies showed positive results (Additional Files 1 and 3).

\section{Risk of bias within and across studies}

Risk of bias within and across RCTs is presented in Table 3. Most RCTs had a high risk of bias. Table 4 shows the risk of bias within and across quasiexperimental studies. There was a consistent pattern of moderate risk at pre-intervention, post-intervention and within studies.

\section{Discussion}

In this review, we have reported on studies published in peer-reviewed journals that investigated falls prevention interventions for community-dwelling older people in Mainland China. We focused on reporting the types of interventions used as well as the components of falls prevention interventions. Although there are a growing number of studies in the past two decades focusing on this emerging public health issue in Mainland China, we identified a lack of research focused on rural areas. In addition, the quality of the evidence was generally poor, and studies did not address scalability and sustainability of interventions.

There were no studies identified prior to the year of 2000. Most studies were conducted after the year of 2011 and this aligns with the Chinese national policy development in relation to older people's health management. The earliest policy attaching importance to falls prevention was the National Essential Public Health Service Package Specification (2009 Edition) issued by the National Health Committee on December 10, 2009 [118]. This policy specified older people's health management as a universal health service provided to all age-eligible people. In addition, since 2009 there have been more than 22 relevant policies issued by Chinese ministries or departments affiliated to the State Council. Over the past decade, four national planning policies have been formulated by the Chinese central government to address the growing needs of the ageing population [119-122]. Of note, falls prevention for older people was specifically assigned as an important task in two policies, the 13th Five-Year Plan for Healthy Ageing (2016-2020) and Healthy China 2030 [121, 122]. There is evidence in the published studies of a strong alignment between the number of studies and the development of these policies. This suggests the prevention of falls has been identified by leading government agencies as a national key priority to achieving healthy ageing.

Nearly $50 \%$ of the studies were implemented in high socioeconomic urban areas. This may be explained by the local government in these areas providing researchers with better funding support to conduct falls prevention research, or, the higher education and health literacy levels of residents in urban areas providing researchers with greater access to, and recruitment of, participants in these areas compared to rural settings. Even when considering the change in population distribution between urban and rural areas since 2011 [123], the proportion of studies investigating community-based falls prevention interventions in rural areas was still disproportionately low given the rural population. This could imply that the impact of falls prevention interventions for community-dwelling older people was limited to the developed regions. Significant health inequalities might therefore occur in falls prevention services provided for community-dwelling older people in more rural provinces in Mainland China, particularly for resource-restricted areas.

In this review, we found falls prevention programs were predominately multiple component interventions, with a preferred combination of knowledge/education, exercise, environment/assistive technology and medication components. However, without taking the underlying risk of falling into consideration, the combination of interventions reported to be effective in studies investigating multiple component interventions might not be successfully applied to all older people in Mainland China due to differences in community settings. Additional evidence is required to demonstrate the effectiveness of these interventions in different community settings. Multifactorial interventions were recommended in the falls prevention guidelines [124-126], but they have not been widely implemented in the Chinese community settings. This result was consistent with the findings from Hopewell's review that the lack of evidence of multifactorial interventions may be explained by the complexity of delivering these interventions, particularly with limited financial and supporting resources [10].

Among all intervention components, the top three frequently used components were distributing written materials, delivering lectures and providing gait, balance and functional training. The first two components were rarely implemented as standalone interventions. They 
Table 3 Risk of bias within and across RCTs

\begin{tabular}{|c|c|c|c|c|c|c|c|}
\hline ID & Authors & Selection bias & Performance bias & Detection bias & Attrition bias & Reporting bias & Within study \\
\hline 1 & Li W, et al. [18] & Unclear & High & Unclear & High & High & High \\
\hline 2 & Duan F, et al. [24] & High & Unclear & Unclear & Low & Low & Unclear \\
\hline 3 & Deng YN, et al. [25] & Unclear & High & Unclear & Low & Low & High \\
\hline 4 & Zhang C, et al. [30] & Unclear & Unclear & Unclear & Low & Low & Unclear \\
\hline 5 & Wang YH, et al. [31] & Unclear & High & High & Unclear & High & High \\
\hline 6 & Feng MM, et al. [32] & Low & High & Low & Low & High & High \\
\hline 7 & Mao XR, et al. [33] & Low & Unclear & Unclear & Low & High & High \\
\hline 8 & Zhang CG, et al. [35] & Unclear & High & Unclear & Low & Low & High \\
\hline 9 & Xie Y, et al. [36] & Unclear & Unclear & Unclear & Low & Low & Unclear \\
\hline 10 & Liu YC, et al. [37] & High & High & Unclear & High & Low & High \\
\hline 11 & Yang SL, et al. [40] & Unclear & Unclear & Unclear & Low & Low & High \\
\hline 12 & Tang LJ, et al. [41] & High & High & Unclear & Low & High & High \\
\hline 13 & Zheng WJ, et al. [42] & Unclear & Unclear & Unclear & Low & Low & Unclear \\
\hline 14 & Xie XF, et al. [43] & High & Unclear & Unclear & Low & Low & High \\
\hline 15 & Ren HJ, et al. [44] & Unclear & Unclear & Unclear & Unclear & Low & Unclear \\
\hline 16 & Liu XY, et al. [45] & Unclear & High & Unclear & Low & Low & High \\
\hline 17 & Zhan JH, et al. [46] & Unclear & High & Unclear & Low & Low & High \\
\hline 18 & Guan SH, et al. [47] & Unclear & High & Unclear & Low & Low & High \\
\hline 19 & Li YL, et al. [48] & High & High & Unclear & Low & Low & High \\
\hline 20 & Liu LD, et al. [49] & Unclear & High & Unclear & Low & Low & High \\
\hline 21 & Zhao D, et al. [52] & Low & Unclear & Unclear & Low & High & High \\
\hline 22 & Zhou LL, et al. [53] & Unclear & Unclear & Unclear & Unclear & High & High \\
\hline 23 & You MY, et al. [54] & High & Unclear & Unclear & Low & Low & High \\
\hline 24 & Ou YB, et al. [55] & Unclear & High & Low & Low & Low & High \\
\hline 25 & Tao WY, et al. [56] & Unclear & Unclear & Unclear & Low & Low & Unclear \\
\hline 26 & Deng XW, et al. [57] & High & Unclear & Unclear & Unclear & High & High \\
\hline 27 & Xiao Y, et al. [58] & Unclear & High & Unclear & Low & Low & High \\
\hline 28 & Liu X, et al. [59] & Unclear & Unclear & Unclear & Low & Low & High \\
\hline 29 & Ji YQ, et al. [60] & Unclear & High & Unclear & Low & Low & High \\
\hline 30 & Zhang RL, et al. [61] & High & High & Unclear & High & Low & High \\
\hline 31 & Li W, et al. [62] & High & High & Unclear & Unclear & Low & High \\
\hline 32 & Chen CF, et al. [63] & Unclear & Unclear & Unclear & Low & Low & Unclear \\
\hline 33 & Zhang J, et al. [64] & Low & Unclear & Unclear & Low & Low & Low \\
\hline 34 & Mou YD, et al. [65] & High & Unclear & Unclear & Low & Low & High \\
\hline 35 & Ma HX, et al. [66] & High & Unclear & Unclear & Low & Low & High \\
\hline 36 & Li DY, et al. [67] & High & Unclear & Unclear & Unclear & High & High \\
\hline 37 & Lu HL, et al. [69] & Unclear & Unclear & High & Unclear & High & High \\
\hline 38 & Liang YY, et al. [70] & Unclear & High & Unclear & Low & Low & High \\
\hline 39 & Li J, et al. [71] & Unclear & Unclear & Unclear & Low & Low & Unclear \\
\hline 40 & Li H, et al. [72] & Unclear & High & Unclear & Low & Low & High \\
\hline 41 & Li J, et al. [73] & Unclear & High & Unclear & Low & Low & High \\
\hline 42 & Zeng $Y Y$, et al. [77] & Unclear & High & Unclear & Low & Low & High \\
\hline 43 & Yuan Y, et al. [78] & Unclear & Unclear & Unclear & Low & Low & Unclear \\
\hline 44 & Wang JY, et al. [79] & Unclear & High & Unclear & Low & Low & High \\
\hline
\end{tabular}


Table 3 Risk of bias within and across RCTs (Continued)

\begin{tabular}{|c|c|c|c|c|c|c|c|}
\hline ID & Authors & Selection bias & Performance bias & Detection bias & Attrition bias & Reporting bias & Within study \\
\hline 45 & Qiu X, et al. [80] & Unclear & Unclear & Unclear & Low & Low & High \\
\hline 46 & Liao SH, et al. [81] & Low & Low & Unclear & Low & Low & Unclear \\
\hline 47 & Wu YQ, et al. [82] & Unclear & High & Unclear & Low & Low & High \\
\hline 48 & Yun FX, et al. [83] & Unclear & High & Unclear & Low & Low & High \\
\hline 49 & Tao YL, et al. [85] & Unclear & High & Unclear & Low & Low & High \\
\hline 50 & Meng FY, et al. [86] & Unclear & High & Unclear & Unclear & Low & High \\
\hline 51 & Wang DZ, et al. [87] & Unclear & High & Unclear & Unclear & Low & High \\
\hline 52 & Tian YQ, et al. [88] & Unclear & High & Unclear & Low & Low & High \\
\hline 53 & Yang XM, et al. [89] & High & High & Unclear & Low & Low & High \\
\hline 54 & LanLi R, et al. [90] & High & High & Unclear & Low & Low & High \\
\hline 55 & Ba HB, et al. [91] & Unclear & High & Unclear & Low & Low & High \\
\hline 56 & Wu H, et al. [92] & Unclear & High & Unclear & Low & Low & High \\
\hline 57 & Nu QL, et al. [93] & Unclear & High & Unclear & Low & Low & High \\
\hline 58 & Yang Z, et al. [94] & Unclear & Unclear & Unclear & Low & Low & Unclear \\
\hline 59 & Hu ZJ, et al. [95] & High & Unclear & Unclear & Low & Low & High \\
\hline 60 & Qiu YY, et al. [97] & Unclear & High & Unclear & Low & Low & High \\
\hline 61 & Wu ML, et al. [98] & Unclear & High & Unclear & Low & Low & High \\
\hline 62 & Yang SL, et al. [99] & Unclear & High & Unclear & Low & Low & Unclear \\
\hline 63 & Cai YB, et al. [100] & Unclear & High & Unclear & Unclear & Low & High \\
\hline 64 & Yang JY, et al. [101] & Unclear & High & Unclear & Unclear & Low & High \\
\hline 65 & Fan XY, et al. [103] & Unclear & Unclear & Unclear & Low & Low & Unclear \\
\hline 66 & Gao R, et al. [104] & High & High & Unclear & Low & Low & High \\
\hline 67 & Tang F, et al. [105] & High & High & Unclear & Unclear & Low & High \\
\hline 68 & Hong D, et al. [106] & Unclear & High & Unclear & Low & Low & High \\
\hline 69 & Zhao Y, et al. [108] & Unclear & High & Unclear & Unclear & Low & High \\
\hline 70 & Wang K, et al. [109] & Low & Unclear & Unclear & Low & Low & Low \\
\hline 71 & Xia QH, et al. [110] & Unclear & Unclear & Unclear & Low & Low & Unclear \\
\hline 72 & Zhuang J, et al. [111] & Low & Unclear & Unclear & Unclear & Low & Unclear \\
\hline 73 & Liu XH, et al. [113] & High & Unclear & Unclear & Low & High & High \\
\hline 74 & Lv B, et al. [114] & Unclear & Unclear & Unclear & Low & Low & Unclear \\
\hline 75 & Gao ML, et al. [115] & Unclear & Unclear & Low & Low & Unclear & Unclear \\
\hline 76 & Deng XQ, et al. [116] & Unclear & Unclear & High & Unclear & High & High \\
\hline 77 & Wu X, et al. [117] & Unclear & Unclear & Unclear & Low & Low & Unclear \\
\hline \multicolumn{2}{|c|}{ Overall across studies } & Unclear & Unclear & Unclear & Low & Low & High \\
\hline
\end{tabular}

usually acted as an integral part of the multiple component or multifactorial falls prevention bundles. Gait, balance and functional training was frequently adopted in all three types of falls prevention interventions, were found to be effective. This finding was consistent with previous studies $[8,9,13]$. In contrast, psychological, social environment, management of urinary incontinence, fluid or nutrition therapy, and surgery interventions were rarely or never used in all studies. There was also uncertain evidence of their effectiveness from the previous research. Their effectiveness in the Chinese community settings might be explored in the future.

Most studies reported two or more fall-related outcomes. Injuries, a harder outcome to measure, were rarely reported in the identified studies, but improved awareness after publicity of falls prevention information and education was reported in half of all 101 studies. Due to the large variation in outcome measures, a quantitative synthesis of the effect size, precision and variance of positive results reported in all selected studies was 
Table 4 Risk of bias within and across quasi-experimental studies

\begin{tabular}{|c|c|c|c|c|c|}
\hline ID & Authors & Pre-intervention & At-intervention & Post-intervention & Within study \\
\hline 1 & Shi J, et al. [17] & Low & Unclear & Low & Moderate \\
\hline 2 & Yan Q, et al. [19] & Moderate & Low & Moderate & Moderate \\
\hline 3 & Yan YY, et al. [20] & Low & Low & Moderate & Moderate \\
\hline 4 & Zhuang J, et al. [21] & Moderate & low & low & Moderate \\
\hline 5 & Zhang LL, et al. [22] & Low & Low & Moderate & Moderate \\
\hline 6 & Wan QP, et al. [23] & Low & Unclear & Moderate & Moderate \\
\hline 7 & Sun XL, et al. [26] & Serious & Low & Unclear & Serious \\
\hline 8 & GuLan DM, et al. [27] & Moderate & Low & Serious & Moderate \\
\hline 9 & Wang J, et al. [28] & Low & Low & Serious & Serious \\
\hline 10 & Ma CH, et al. [29] & Serious & Low & Unclear & Serious \\
\hline 11 & Jiang CX, et al. [34] & Low & Unclear & Moderate & Moderate \\
\hline 12 & Liu XY, et al. [38] & Moderate & Low & Low & Moderate \\
\hline 13 & Chen LP, et al. [39] & Moderate & Unclear & Moderate & Moderate \\
\hline 14 & Zhang $X_{\text {, et al. [50] }}$ & Moderate & Low & Moderate & Moderate \\
\hline 15 & Mei D, et al. [51] & Low & Low & Moderate & Moderate \\
\hline 16 & Zhou LW, et al. [68] & Unclear & Unclear & Moderate & Moderate \\
\hline 17 & Jiang CY, et al. [74] & Moderate & Low & Moderate & Moderate \\
\hline 18 & Guo YF, et al. [75] & Low & Low & Moderate & Moderate \\
\hline 19 & Guo YF, et al. [76] & Moderate & Low & Low & Moderate \\
\hline 20 & Hu SM, et al. [84] & Low & Low & Moderate & Moderate \\
\hline 21 & Gui RL, et al. [96] & Moderate & Low & Moderate & Moderate \\
\hline 22 & Guo LY, et al. [102] & Moderate & Low & Moderate & Moderate \\
\hline 23 & Liu M, et al. [107] & Moderate & Unclear & Low & Moderate \\
\hline 24 & Cao ZJ, et al. [112] & Low & Unclear & Low & Moderate \\
\hline \multicolumn{2}{|c|}{ Overall across studies } & Moderate & Low & Moderate & Moderate \\
\hline
\end{tabular}

not performed, however our findings are consistent with the findings of other published literature [10-12]. None of the included studies provided a registered protocol and most were conducted with a small sample size. In addition, the level of bias assessed in these studies was high primarily due to the lack of information available within the randomized and non-randomized studies to assess bias. Despite all selected studies showed positive results, the interpretation of these results should be used with caution. Therefore, the high-quality evidence is currently not available to guide scale-up of the falls prevention interventions described in the studies. Thus, there is an opportunity to undertake an evaluation of a rigorously-designed, large-scale falls prevention program for community-dwelling older people in Mainland China.

This review synthesized the main characteristics of studies evaluating the effects of falls prevention interventions in community-dwelling older people in Mainland China. Three recommendations for future falls prevention research in Mainland China are: one, community-based falls prevention interventions need to be evaluated in rural settings; two, multifactorial interventions that include individual risk assessment and personalised interventions need to be investigated; three, the effectiveness of poorly utilised interventions, such as psychological, social environment, management of urinary incontinence, fluid or nutrition therapy and surgery, need further exploration and investigation for older people in Mainland China.

\section{Strengths and limitations}

To our knowledge, this was the first review to systematically summarise the key features of studies delivering falls prevention interventions for community-dwelling older people living in Mainland China. This review provides an overview of the essential characteristics, interventions, and outcomes of studies conducted in community settings of Mainland China since 1990. Two limitations of this review have been identified Firstly, falls prevention interventions for community-dwelling older people in Mainland China might not have been identified by the search strategy since some interventions may be reported in non-peer-reviewed 
literature, e.g., reports, thesis or conference papers, and the characteristics of these interventions may differ from those reflected in this review. Secondly, a direct comparison and pooled analysis could not be performed due to the heterogeneity of outcome measurements.

\section{Conclusion}

As population ageing has intensified, falls have been recognised as a preventable, but complex, health issue amongst older people. Community-based falls prevention interventions have been delivered to older people in different provinces of Mainland China during the last two decades. However, there is still a lack of evidence for robust, sustainable and scalable falls prevention interventions that could be integrated into policy and the health system in Mainland China. There is an urgent need to conduct welldesigned studies to produce high-quality evidence for the most effective falls prevention interventions in Mainland China. The findings of this study will also potentially provide evidence base for other low- and middle-income countries with a rapidly ageing population but underequipped health system to combat falls.

\section{Supplementary information}

Supplementary information accompanies this paper at https://doi.org/10. 1186/s12913-020-05645-0.

Additional file 1. The details of all eligible studies

Additional file 2. The types, categories and components of falls prevention of all eligible studies.

Additional file 3. The outcome types of all eligible studies.

Additional file 4. The example of detailed search strategy.

\section{Abbreviations}

RCTs: Randomised controlled trials; ROBINS-I: Risk of Bias in Non-Randomized Studies-of Interventions; GBD: Global Burden of Disease; DALYs: Disabilityadjusted life-years; PRISMA: Preferred Reporting Items for Systematic Review and Meta-analysis; Embase: Excerpta; Medica Database; CNKl: Chinese National Knowledge Infrastructure; CMBdisc: China Biology Medicine disc

\section{Acknowledgements}

We would like to thank all people of this study for interesting and informative discussions.

\section{Authors' contributions}

All authors have contributed to the production of this review. PY, YL and MT designed the study. PY, YL, JZ, KP, XP, YS, and SX participated in the systematic review process. YE and LD were involved in the interpretation of the data. PY and YL drafted the manuscript. EA, RI, LK and MT provided critical comments on drafts of the manuscript. All authors participated in the review of the manuscript, read and approved the final manuscript.

\section{Funding}

Pengpeng Ye is supported by the Dr. John Yu Fellowship from the George Institute for Global Health and Tuition Fee Scholarships from University of New South Wales. Yishu Liu is supported by the University International Postgraduate Award from University of New South Wales. Elizabeth Armstrong is supported by an Australian Government Research Training Program (RTP) Scholarship. The funders exercised no control or influence over the design of the study or the collection, analysis, and interpretation of data or during the writing of the manuscript.
Availability of data and materials

All data analysed or produced as a result of this review are included in the main file and Additional files.

Ethics approval and consent to participate

Not applicable.

\section{Consent for publication}

Not applicable.

\section{Competing interests}

All authors declare no competing interests.

\section{Author details}

${ }^{1}$ The George Institute for Global Health, University of New South Wales, PO Box M201, Missenden Road, Sydney, NSW 2050, Australia. ${ }^{2}$ National Centre for Non-Communicable Disease Control and Prevention, Chinese Centre for Disease Control and Prevention, Beijing, China. ${ }^{3}$ The George Institute for Global Health at Peking University Health Science Centre, Beijing, China. ${ }^{4}$ School of Population Health, University of New South Wales, Sydney, Australia. ${ }^{5}$ School of Public Health, The University of Sydney, Sydney, Australia. ${ }^{6}$ School of Public Health, Peking University Health Science Centre, Beijing, China. ${ }^{7}$ Falls Balance and Injury Research Centre, Neuroscience Research Australia, Randwick, Australia. ${ }^{8}$ School of Optometry and Vision Science, University of New South Wales, Sydney, Australia.

Received: 24 March 2020 Accepted: 12 August 2020

Published online: 28 August 2020

\section{References}

1. WHO. WHO global report on falls prevention in older age. Geneva: World Health Organization; 2007.

2. WHO. World report on ageing and health. Geneva: World Health Organization; 2015.

3. Kyu HH, Abate D, Abate KH, Abay SM, Abbafati C, Abbasi N, et al. Global, regional, and national disability-adjusted life-years (DALYs) for 359 diseases and injuries and healthy life expectancy (HALE) for 195 countries and territories, 1990-2017: a systematic analysis for the global burden of disease study 2017. Lancet. 2018;392(10159):1859-922.

4. Leilei D, Pengpeng Y, Haagsma JA, Ye J, Yuan W, Yuliang E, et al. The burden of injury in China, 1990-2017: findings from the global burden of disease study 2017. Lancet Public Health. 2019;4(9):e449-e61.

5. Cumming RG. Intervention strategies and risk-factor modification for falls prevention: a review of recent intervention studies. Clin Geriatr Med. 2002; 18(2):175-89.

6. Costello E, Edelstein JE. Update on falls prevention for community-dwelling older adults: review of single and multifactorial intervention programs. J Rehabil Res Dev. 2008;45(8):1135-52.

7. Gillespie LD, Robertson MC, Gillespie WJ, Sherrington C, Gates S, Clemson $L M$, et al. Interventions for preventing falls in older people living in the community. Cochrane Database Syst Rev. 2012;9:CD007146.

8. Kendrick D, Kumar A, Carpenter H, Zijlstra GA, Skelton DA, Cook JR, et al. Exercise for reducing fear of falling in older people living in the community. Cochrane Database Syst Rev. 2014;11:CD009848.

9. El-Khoury F, et al. The effect of fall prevention exercise programmes on fall induced injuries in community dwelling older adults: systematic review and meta-analysis of randomised controlled trials. BMJ. 2013;347:f6234.

10. Hopewell S, Adedire O, Copsey BJ, Boniface GJ, Sherrington C, Clemson L, et al. Multifactorial and multiple component interventions for preventing falls in older people living in the community. Cochrane Database Syst Rev. 2018;7:CD012221.

11. Hill KD, Suttanon P, Lin SI, Tsang WWN, Ashari A, Hamid TAA, et al. What works in falls prevention in Asia: a systematic review and meta-analysis of randomized controlled trials. BMC Geriatr. 2018;18(1):3.

12. Cheng $P$, Tan $L$, Ning $P$, Li L, Gao Y, Wu Y, et al. Comparative Effectiveness of Published Interventions for Elderly Fall Prevention: A Systematic Review and Network Meta-Analysis. Int J Environ Res Public Health. 2018;15(3).

13. Sherrington C, Fairhall NJ, Wallbank GK, Tiedemann A, Michaleff ZA, Howard $\mathrm{K}$, et al. Exercise for preventing falls in older people living in the community. Cochrane Database Syst Rev. 2019;1:CD012424. 
14. Moher D, Liberati A, Tetzlaff J, Altman DG. Preferred reporting items for systematic reviews and meta-analyses: the PRISMA statement. BMJ. 2009; 339:b2535.

15. Higgins JPT, Altman DG, Gøtzsche PC, Jüni $P$, Moher D, Oxman AD, et al. The Cochrane Collaboration's tool for assessing risk of bias in randomised trials. BMJ. 2011;343:d5928.

16. Sterne JA, Hernán MA, Reeves BC, Savović J, Berkman ND, Viswanathan M, et al. ROBINS-l: a tool for assessing risk of bias in non-randomised studies of interventions. BMJ. 2016;355:14919.

17. Shi J, QingYao H, Tao YK, Zhou BY, Yu PL, Duan CB, et al. Study on falls incidence and effectiveness of falls intervention programs in communitydwelling older people in Beijing. Zhongguo Lao Nian Xue Za Zhi. 2015; 35(10):2792-4.

18. Li W, Lu XQ, Wang SP, Zhou XP, Zhao HX, Zhang YY. Preventing falls in the elderly. Zhongguo Lao Nian Xue Za Zhi. 2009;29:2268-9.

19. Yan Q, Liu F. The role of safety education and home environment reform in preventing the elderly falls. Zhonghua Hu Li Za Zhi. 2008;43(10):946-7.

20. Yan YY, Li AH, Du AL. Intervention evaluation on fall injuries among community elderly people in Luoyang, Henan Province. Zhongguo Jian Kang Jiao Yu. 2012;28(4):332-3.

21. Zhuang J, Wang JQ, Huang L, Wu YQ, Yu B, Li HJ, et al. The effect of a 3month combined exercise intervention on gait in community-dwelling elderly women. Shenyang Ti Yu Xue Yuan Xue Bao. 2011;30(6):83-6.

22. Zhang LL, Dalal K, Yin MM, Yuan DG, Andrews JY, Wang SM. The KAP evaluation of intervention on fall-induced injuries among elders in a safe community in Shanghai, China. PLoS One. 2012;7(3):e32848.

23. Wan QP, Quan L, Xu W, Long ZX, Zhang GH. Assessment of intervention on falls risk environment among old solitary people at home. Zhongguo Jian Kang Jiao Yu. 2012;28(4):282-5.

24. Duan F. Effects of preventive exercise of falling on the community elderly physical performance. Shandong Ti Yu Xue Yuan Xue Bo. 2011;27(11):59-62.

25. Deng YN. Study on comprehensive nursing intervention of preventing falls in the elderly patients with hypertension and diabetes in community. Yi Xue Xing Xi. 2014;27(12):131-2.

26. Sun $X L$. Home-based elderly strengthens behavior training to prevent falls. Yi Xue Mei Xue Mei Rong. 2015;549.

27. GuLan DM. The investigation on the role of safety education and home environment reform in preventing elderly people from falling. Zhongguo Wu Zhen Xue Za Zhi. 2012;12(1):129.

28. Wang J, Jiang CY. Comprehensive interventions to prevent falls among elderly residents in the community. Hu Li Xue Bao. 2012;19(9B):61-3.

29. $\mathrm{Ma} \mathrm{CH}$. Analysis of nursing measures preventing falls for elderly patients with hypertension in community. Xian Dai Hu Li. 2013;12(10):467-8.

30. Zhang C. Current status investigation of knowledge-attltude-behavior about elderly falls in a high school community of Guangzhou and evaluatlon of the interventlon measures. Xian Dai Yi Yuan. 2013:13(9):152-3.

31. Wang YH, Liu SX. Effects of different health education methods on the pain and falls of elderly people with high-risk osteoporotic bone fractures in the community. Zhongguo Lao Nian Xue Za Zhi. 2014;7:1697-9.

32. Feng MM, Wang XY. Impact of fall prevention exercise intervention on exercise ability of community elderly. Zhongguo Ji Ceng Yi Yao. 2014;21(5): 798-9.

33. Mao XR, Du WJ, Xu YZ, Chen RM, Gong CT, Jiao NN, et al. Effectiveness of comprehensive nursing interventions in preventing falls in elderly patients with hypertension or diabetes in the community. Zhongguo Quan Ke Yi Xue Za Zhi. 2013;16(28):575-8.

34. Jiang CX, Ma XY, Duan LL, Er YL, Zhang XJ, Gao C, et al. Study on effectiveness of falls intervention programs in community-dwelling older people in Shijiazhuang. Zhonghua Ji Bing Kong Zhi Za Zhi. 2017;21(8):7725 .

35. Zhang CG, Yu JM, Cui ZW, Song R. Study on fall intervention of elderly population in urban communities in Xuhui District of Shanghai. Zhi Ye Yu Jian Kang. 2016;32(6):771-3.

36. Xie $Y$. The analysis of comprehensive nursing intervention for the elderly in fall community. Zhong Guo Yi Yao Zhi Nan. 2013;11(5):331-2.

37. Liu YC. Impact of recreational rehabilitation on the fall rate of the elderly in the community. Yi Yao Qian Yan. 2015;5(16):340-1.

38. Liu XY, Yin BX. Application and effect of eight-section brocade exercise therapy in prevention of fall in community elderly. Hu Li Yan Jiu. 2016;30(2): 423-4.
39. Chen LP. Application of non-continuous education methods in the improvement of life quality and prevention of falls in elderly people in community. Qilu Hu li Za Zhi. 2014;20(22):3.

40. Yang SL, Xu GN, Luo W. The effect of complex avoiding obstacles training in preventing falls in old people. Zhongguo Kang Fu Yi Xue Za Zhi. 2012; 27(11):1026-30.

41. Tang $L J$, Yue $C L$, Xiang LC, Wu L, Peng YY. Effects of Otago exercise on fear of falling and balance ability in parkinson's discharge patients. Zhongguo Kang Fu Yi Xue Za Zhi. 2016;31(12):1383-5.

42. Zheng WJ. Related factors analysis and nursing measurement for the community hypertension elderly fall. Zhongguo Chu Ji Wei Sheng Bao Jian. 2015;29(4):121-2.

43. Xie XF, Huang ZH, Zhang HC. Application of community intervention combined with health education in preventing elderly people from falling. Zhongguo Min Zu Min Jian Yi Yao. 2016;25(4):161-2

44. Ren HJ, Hu JP, Li JB, Wang Y, Du XJ, An HY. The effect of the muscle strength training in the secondary prevention of decreased muscle strength for the elderly. Xian Dai Yu Fang Yi Xue. 2015;42(11):2021-4.

45. Liu XY, Gao J, Bai DX, Yang XY, Zhang HY. Study on influence of eightsectioned exercise on falls efficacy in community elderly. Hu Li Yan Jiu. 2015;29(1):90-2.

46. Zhan $\mathrm{JH}$. The effect of family prevention guidance on preventing elderly falls. Yi Xue Xing Xi. 2015;28(9):226

47. Guan SH. Application value of community care in the prevention of falls in elderly people. Shu Li Yi Yao Xue Za Zhi. 2017;30(4):623-4.

48. Li YL. Analysis of the effect of comprehensive nursing care for falls prevention in older people with high blood pressure living in the community. Neimenggu Zhong Yi Yao. 2014;16:137-8.

49. Liu LD. The evaluation of comprehensive nursing intervention in preventing fall of elderly hypertensive or diabetic patients in community. Lin Chuang $Y$ Yao Wen Xian Za Zhi. 2016;3(26):5235.

50. Zhang X, Gong QQ, Yang YH, Ye CS. Assessment of the effect of multifactor assessment and intervention on the prevention of falls among the elderly in the community. Shanghai Yi Yao. 2016:37(16):51-3.

51. Mei D, Jiang J, Wang XF, Liu D. Evaluation of intervention effect of improving environment on falls in the elderly. Shang Hai Yi Xue. 2017;6(4): 14-8

52. Zhao D, Xie T, Guo WQ, Wang Y. The effect of knowing how to prevent people from falling in fear of falling in the community. Zhongguo Lao Nian Yi Xue Za Zhi. 2015:35:4950-2.

53. Zhou LL. Analysis of the related factors and nursing care of falls in elderly patients with hypertension in community. Zhong Guo Yi Yao Zhi Nan. 2016; 14(1):256

54. You MY, Zou GF, Ou HZ. Effect analysis of comprehensive nursing intervention in falls prevention of community senile hypertension or diabetes patients. Zhongguo Xian Dai Yao Wu Ying Yong. 2017;11(6):154-6.

55. Ou YB, Gong YX, Liu D, Luan YM. The application of the fall risk management for the community elderly in the aspects of fall prevention. Zhongguo Kang Fu. 2015;30(4):277-9.

56. Tao WY. Effectiveness of comprehensive nursing interventions in preventing falls in elderly patients with hypertension or diabetes in the community. Yang Sheng Bao Jian Zhi Nan. 2017;52:67-8.

57. Deng XW, Zhou HB, Lei L, Peng J, Feng NP. Efficacy of health education program regarding prevention of falls among community-dwelling elderly adults in Shenzhen City. Zhongquo Jian Kang Jiao Yu. 2018:34(3):174-7.

58. Xiao $Y$. The effectiveness of comprehensive nursing interventions in preventing falls in elderly patients with hypertension or diabetes in the community. Da Jia Jian Kang. 2015;9(3):199-200.

59. Liu X, Wang GX, Lv YX, FengYang J, Wen LZ, Zhou YX. Risk factors and intervention measures of osteoporotic fracture in the elderly. Haerbing Yi Yao. 2017;37(3):221-2

60. Ji YQ. The prevention and nursing methods of preventing falls for community-dwelling older people with hypertension or diabetes. Yi Xue Shi Liao Yu Jian Kang. 2018:94-5.

61. Zhang RL, Zhu LH. Preventive effects of comprehensive nursing care in elderly patients with high risk of osteoporotic fracture. Wenzhou Yi Ke Da Xue Xue Bao. 2018;48(3):225-6.

62. Li W, Wang DX, Jiao LM. Application of continuous intervention in preventing falls after discharge for fracture patients in the community. $\mathrm{Xi}$ Nan Guo Fang Yi Yao. 2018:28(5):485-6. 
63. Chen CF, Xu H, Gu QL, Wu JF. Study on the effect of group baduanjin exercises on fall risk and quality of life of elderly patients. Zhongguo She Qu Yi Shi. 2016;32(28):170-2.

64. Zhang J, Lin C. Balance training for the elderly fall risk intervention observation. Zhongguo Mei Tan Gong Ye Yi Xue Za Zhi. 2016;19(4):518-21.

65. Mou YD. Evaluate the effect of comprehensive nursing interventions to prevent falls in elderly elderly patients with hypertension or diabetes. Zhongguo Bao Jian Ying Yang. 2016;10:199.

66. Ma HX. The effect of comprehensive nursing intervention on prevention of falls in elderly patients with high blood pressure or diabetes in the community. Zhongguo Bao Jian Ying Yang. 2017;5:289.

67. Li DY. The effect of comprehensive nursing intervention on preventing falls in elderly patients with hypertension or diabetes. Zhongguo Di Fang Bing Fang Zhi Za Zhi. 2017;32(5):588

68. Zhou LW. The value of community nursing in the prevention of falls in the elderly. Zhongguo Yao Wu Jing Ji Xue. 2016;6:141-2.

69. Lu HL. Effect of comprehensive nursing care on prevention of falling in old people with high blood pressure or diabetes in community areas. Da Jia Jian Kang. 2017;11(21):244.

70. Liang YY, Tan QN, Gan XY. Impact of comprehensive community intervention on preventing falls in elderly patients with chronic diseases. Qilu Hu li Za Zhi. 2019;25(09):78-90.

71. Li J, Chen $\mathrm{XL}$, Xie YH. The effect of nursing intervention on prevent occurrence of fall in elderly patients with hypertension or diabetes mellitus. Qiqihaer Yi Xue Yuan Xue Bao. 2017;38(9):1112-4.

72. Li H, Jiang WZ, Ma JL, Wang XJ. The evaluation of the effect of power balance exercise on the prevention of falls in the elderly in community. Shanghai Yi Yao. 2016;37(24):52-4.

73. Li J, Liu LL. The effect of community-based comprehensive nursing intervention for falls prevention in elderly patients with hypertension. Shijie Zui Xin Yi Yao Xue Xing Xi Wen Zhai. 2015;15(67):248-9.

74. Jiang CY, Zhang Y. Evaluation of nursing interventions effect on falls in elderly people with moderate or high risk of falls in community. Jie Fang Jun Hu Li Za Zhi. 2017:34(15):10-3.

75. Guo YF, Tian H, Li P, Cao JH, Sun YH, Zhang M. The evaluation on intervention effect of cognition, attitude, and behavior aiming at prevention of the elderly falls at home. Hu Li Yan Jiu. 2017;31(13):1625-7.

76. Guo YF, Li P, Tian H, Cao JH. The investigation and analysis of the effect of balance ability evaluation and individualized intervention on the falls prevention of the elderly in the community. Xinjiang Yi Xue. 2016;46(4):4157.

77. Zeng YY. Effect of comprehensive intervention on reducing fall of community elderly. Hu Li Shi Jian Yu Yan Jiu. 2016;13(18):148-9.

78. Yuan Y, Li B. Community-based fall prevention strategies evaluation for primary osteoporosis in elderly people. Zhongguo Quan Ke Yi Xue Za Zhi. 2019;22(28):3461-3.

79. Wang JY, Zhao QS, Liu XF, He Y, Deng YL, Wang XC. Intervention study on falls among the elderly in Fangshan District in 2016. Zhongguo Man Xing Bing Yu Fang Yu Kong Za Zhi. 2017;25(12):912-4.

80. Qiu X. The effect of comprehensive nursing intervention in preventing falls in elderly patients with hypertension or diabetes in community. Xin Xue Guan Fang Bing Zhi Shi. 2018;7:3-5.

81. Liao SH. The effect of comprehensive nursing interventions on preventing falls in elderly patients with chronic diseases in the community. Haerbing Yi Yao. 2018;38(2):192-3.

82. Wu YQ. Nursing interventions for safe living environment of elderly patients with hypertension and diabetes in community. Yi Xue Mei Xue Mei Rong. 2019;28(3):147-8

83. Yun FX, Peng YQ, Weng XQ. Effect of table tennis on muscle strength of lower extremities and static balance of the elderly. Hubei Ti Yu Ke Ji. 2017; 36(3):233-5

84. Hu SM, Yang YY, Li XL, Lin HN. The analysis of risk factors for falls in the elderly with the family doctor contract service model and the evaluation of intervention effect. Lin Chuang He Li Yong Yao. 2018;11(4A):142-4.

85. Tao YL, Chen HJ, Ly F, Lin ZY, Liao QF, Chen YP. Application of four-party co-nursing intervention to community-dwelling elderly people at high riks of falls. Hu Li Xue Za Zhi. 2019;34(13):88-91.

86. Meng FY, Chen X, Wang Y, Wu Y. Effect of reality-based functional gait training on gait characteristics and fear of fall in community-dwelling elderly convalescent stroke patients. Hu Li Xue Bao. 2018;25(19):1-4.
87. Wang DZ. Risk factors and preventive measures of falling in the community elderly. Jian Kang Zhi You. 2019;104.

88. Tian YQ. Application of community sports interventions in preventing falls in the elderly. Jian Kang Zhi You. 2019:196-7.

89. Yang XM. Effectiveness of comprehensive nursing interventions to prevent falls in elderly patients with hypertension or diabetes. Jian Kang Bi Du. 2019; 5:156

90. LanLi R. Analysis on the effect of nursing care in the home environment safety of elderly patients with hypertension and diabetes in community. Tang Niao Bing Xin Shi Jie. 2019:103-4.

91. Ba HB, Chen L. Balance ability, muscle strength, and neuromuscular response of lower extremities in older adult women during backward exercise. Zhongguo Zu Zhi Gong Cheng Yan Jiu. 2018;22(20):3161-6.

92. Wu H, Ma YY, Qu FF. The effectiveness of comprehensive nursing interventions in preventing falls in elderly patients with hypertension or diabetes in the community. Zhong Xi Yi Jie He Xue Guan Za Zhi. 2019;7(10): 136.

93. Nu QL. The effect of comprehensive nursing intervention to prevent the falls of older peopel with hypertension or diabetes living in the community. Zhong Xi Yi Jie He Xue Guan Za Zhi. 2018;6(1):103-4.

94. Yang Z. Effect of progressive core strength training on balance for old adults. Zhongguo Kang Fu Li Lun Yu Shi Jian. 2019;25(7):836-9.

95. Hu ZJ. A study on the effect of health education on preventing falls in older people in the urban communities. Yi Xue Xing Xi. 2019;32:97-8.

96. Gui RL, Qi MP, Zhang PJ. Evaluation of the effect of narrative nursing combined with micro-video missionary on the prevention of falls in the elderly in the community. Hu Li Yan Jiu. 2019:164-5.

97. Qiu YY, Cui M. A study on mechanism of Taijiquan exercises in senile people to prevent fall. Hu Li Yan Jiu. 2008;22(2):490-1.

98. Wu ML, Zhang XQ, Luo H, Du XL. Influence of different health education models on prevention of fall of the elderly in community. Hu Li Yan Jiu. 2012;26(1B):186-7.

99. Yang SL, Luo W, Wang HM. Analysis of comprehensive nursing intervention for falls prevention in the elderly living in the community. Zhongguo Lao Nian Xue Za Zhi. 2012;32(23):5281-2.

100. Cai YB. The effect of nursing intervention for falls prevention among elderly adults in rural community. Yi Xue Xing Xi. 2014;27(5):375

101. Yang JY. Community care to prevent falls in the elderly. Yi Xue Xing Xi. 2014;27(10):203.

102. Guo LY, Guo BF. Study on comprehensive nursing intervention for falls prevention of elderly hypertensive patients in community. Shijie Zhong Yi Yao. 2015;10:1645

103. Fan $X Y$. The application of community intervention and health education in preventing the fall of the elderly. Shijie Zui Xin Yi Yao Xue Xing Xi Wen Zhai. 2016;16(60):206-7.

104. Gao R. The effect of community nursing intervention on reducing falls in the elderly. Yi Xue Xing Xi. 2016;29(14):159-60.

105. Tang F, Xu JL, Liang HX. Intervention study on prevention of fracture in high-risk population of elderly patients with osteoporosis in a community of Shanghai. Zhongguo She Qu Yi Shi. 2018;34(35):159-61.

106. Hong D, Xu J, Lin M, Zhang JY, Ye HH. The effects of simplified tai chi exercise on fear of falling among the elderly in communities. Zhonghua $\mathrm{Hu}$ Li Za Zhi. 2018:53(10):1224-9.

107. Liu M, Zhang Q, Hu L, Su QY, Li M, Li Y. The exploration of communitybased support for the risk of falling of older people living alone in Kunming, Yunan Province. Xin Li Yue Kan. 2019;14(11):230-1.

108. Zhao Y. Impact of community aged care programs on cognitive function and falls risk in the elderly. Jian Kang Bi Du. 2019;8:193-4.

109. Wang K, He YP, Wang Y. Effect of Therapeutic Recreation on Prevention of Community Elders Falling. Zhongguo Kang Fu Li Lun Yu Shi Jian. 2009;15(9): $882-3$.

110. Xia QH, Jiang Y, Niu CJ, Tang CX, Xia ZL. Effectiveness of a communitybased multifaceted fall-prevention intervention in active and independent older Chinese adults. Injury Prev. 2009;15(4):248-51.

111. Zhuang J, Huang L, Wu Y, Zhang $Y$. The effectiveness of a combined exercise intervention on physical fitness factors related to falls in community-dwelling older adults. Clin Interv Aging. 2014;9:131-40.

112. Cao ZJ, Wang SM, Zheng WJ, Guo JN, Qu SX. Evaluation of the Communitybased exercise intervention on fall among the elderly. Zhongguo Lao Nian Bao Jian Yi Xue. 2014;12(3):33-7. 
113. Liu XH. Nursing interventions for safe living environment of elderly patients with hypertension and diabetes in community. Jia Ting Yi Yao. 2017:179-80

114. Lv B. Effect of community intervention and health education on falls prevention in the elderly. Zhongguo Bao Jian Ying Yang. 2016;10:361.

115. Gao ML, Song YT. Effect of personalized intervention after falling down on life quality improvement in elderly people. Zhonguo Yi Yao. 2016;11(7): 1084-6.

116. Deng XQ. Effectiveness of comprehensive nursing interventions to prevent falls in elderly patients with hypertension or diabetes. Yi Liao Zhuang Bei. 2017;30(16):180-1.

117. Wu X, Xue WG, Fang J, Wang JX, Fan XL, Ma HX, et al. Baduanjing of movements on the risk of falls in community elderly people for 60 cases. Zhongguo Zhong Yi Yao Xian Dai Yuan Cheng Jiao Yu. 2017;3(251):106-9.

118. Ministry of Health of China. National Essential Public Health Service Package Specification (2009 Edition). Available from http://www.chinalawedu.com/ falvfagui/fg22598/506257.shtml (accessed 2 Oct 2019).

119. State Council of China. Notice on Printing and Distributing the 12th FiveYear Development Plan on Work Concerning Older People (2011-2015). Available from http://www.gov.cn/zwgk/2011-09/23/content_1954782.htm. (accessed 2 Oct 2019).

120. State Council of China. Notice on Printing and Distributing the 13th Five-Year National Development Plan on Work Concerning Older People and the Construction of Aged Care System (2016-2020). Available from http://www.gov.cn/zhengce/content/2017-03/06/content_5173930.htm. (accessed 2 Oct 2019).

121. National Health Committee of China. Notice on Printing and Distributing the 13th Five-Year Plan for Healthy Ageing (2016-2020). Available from http://www.nhc.gov.cn/jtfzs/jlgf/201703/63ce9714ca164840be76b362856a6 c5f.shtml. (accessed 2 Oct 2019).

122. State Council of China. Notice on Printing and Distributing the Healthy China 2030. Available from http://www.gov.cn/zhengce/2016-10/25/ content_5124174.htm. (accessed 2 Oct 2019).

123. Pan JH, Wei HK, Li YJ, et al. China Urban Development Report No.5. Beijing: Social Science Academic Press (China), 2012.

124. National Institute for Health and Clinical Excellence. Falls in older people: assessing risk and prevention 2013 [CG161]. www.nice.org.uk/guidance/ cg161/chapter/1recommendations (accessed 27 September 2018)

125. ACSQH 2009 Australian commission on safety and quality in healthcare. Preventing falls and harm from falls in older people. Best practice guidelines for Australian community care 2009. www.safetyandquality. gov.au/wp-content/uploads/2012/ 01/guidelines-COMM.Pdf. (accessed 27 September 2018).

126. Panel on Prevention of Falls in Older Persons, American Geriatrics Society and British Geriatrics Society. Summary of the updated American Geriatrics Society/British Geriatrics Society clinical practice guideline for prevention of falls in older persons. J Am Geriatr Soc. 2011;59(1):148-57.

\section{Publisher's Note}

Springer Nature remains neutral with regard to jurisdictional claims in published maps and institutional affiliations.

\section{Ready to submit your research? Choose BMC and benefit from:}

- fast, convenient online submission

- thorough peer review by experienced researchers in your field

- rapid publication on acceptance

- support for research data, including large and complex data types

- gold Open Access which fosters wider collaboration and increased citations

- maximum visibility for your research: over $100 \mathrm{M}$ website views per year

At $\mathrm{BMC}$, research is always in progress.

Learn more biomedcentral.com/submissions 